\title{
Wrinkling of Ultrathin Polymer Films
}

Rui Huang ${ }^{1}$, Christopher M. Stafford ${ }^{2}$, and Bryan D. Vogt ${ }^{2}$

${ }^{1}$ Department of Aerospace Engineering and Engineering Mechanics, University of Texas, Austin, TX, 78712

${ }^{2}$ Polymers Division, National Institute of Standards and Technology, Gaithersburg, MD, 20899

\begin{abstract}
This paper presents a bilayer model to account for surface effects on the wrinkling of ultrathin polymer films. Assuming a surface layer of finite thickness, effects of surface properties on the critical strain, the equilibrium wavelength, and the wrinkle amplitude are discussed in comparison with conventional analysis. Experimental measurements of wrinkling in polymer films with thickness ranging from $200 \mathrm{~nm}$ to $5 \mathrm{~nm}$ are conducted. The bilayer model provides a consistent understanding of the experiments that deviate from conventional analysis for thickness less than $30 \mathrm{~nm}$. A set of empirical surface properties is deduced from the experimental data.
\end{abstract}

\section{INTRODUCTION}

Mechanics was established long ago for wrinkling of elastic faces in structural sandwich panels [1] and has been revisited recently [2-5] for nonlinear analysis and inelastic behavior in thin films. For an elastic film on an elastic substrate (Figure 1), a critical compressive stress exists, beyond which an equilibrium wrinkled state can be determined from an energetic analysis that accounts for elastic strain energy in the film and in the substrate. Surface energy is considered negligible in the conventional analysis. However, as the film thickness reaches the nanometer scale, surface energy as well as other surface effects can become important [6].

Recently, a wrinkle-based metrology was developed to measure elastic properties of thin films [7]. For ultrathin polymer films (thickness less than $30 \mathrm{~nm}$ ), the measured wrinkle wavelengths deviate from the conventional solution, and the deduced elastic modulus decreases with decreasing film thickness. In addition, the measured wrinkle amplitudes also differ significantly from the conventional analysis. These experimental results raise a fundamental question as to what are the physical origins for the deviations, in particular, the thicknessdependence of the deduced elastic modulus in the ultrathin films. This paper proposes a bilayer model to account for the surface effect on wrinkling that explains the apparent size effect.

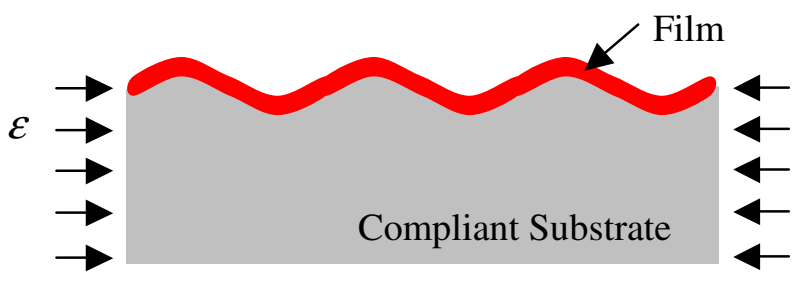

(a)

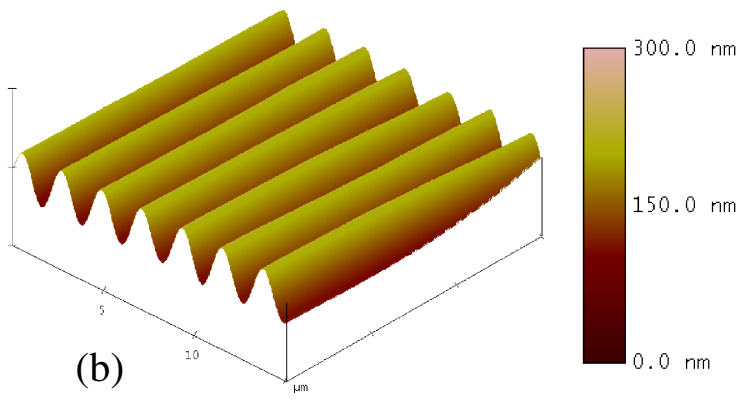

Figure 1. (a) Schematic of an elastic film wrinkling on a compliant substrate; (b) AFM height image of a wrinkled PS film $\left(M_{w}=1800 \mathrm{~kg} / \mathrm{mol}, h=40.4 \mathrm{~nm}\right)$ on a PDMS substrate. 


\section{EXPERIMENTS}

Thin films of polystyrene (PS) were spin-coated from dilute toluene solutions onto polished silicon wafers. Film thickness was varied from $200 \mathrm{~nm}$ to $5 \mathrm{~nm}$ as determined by x-ray reflectivity. Films were then transferred onto polydimethylsiloxane (PDMS) substrates and mounted to a home-built apparatus [8] in order to apply a uniaxial compressive strain. The magnitude of the strain was constant $(2.5 \%)$ for all films in this study. Wrinkling of the PS films was imaged by tapping mode atomic force microscopy (AFM), from which the wavelengths and amplitudes were measured. Figure 1(b) shows an AFM height image of wrinkling in a PS film having a thickness of $40.4 \mathrm{~nm}$. Figure 2(a) shows the measured wrinkle wavelength as a function of film thickness. Two different molecular masses of PS were examined $\left(M_{w}=114 \mathrm{~kg} / \mathrm{mol}\right.$ and $1800 \mathrm{~kg} / \mathrm{mol}$ ), both well above the entanglement molecular mass of PS $\left(M_{e}=19 \mathrm{~kg} / \mathrm{mol}\right)$. Figure 2(b) shows the wrinkle amplitude as a function of film thickness for the PS films with higher molecular mass. The elastic modulus of the PDMS substrate was measured beforehand using a traditional tensile test and was found to be $1.33 \mathrm{MPa}$ for the substrate of the lower molecular mass PS films and 1.75 MPa for the substrate of the higher molecular mass PS films.
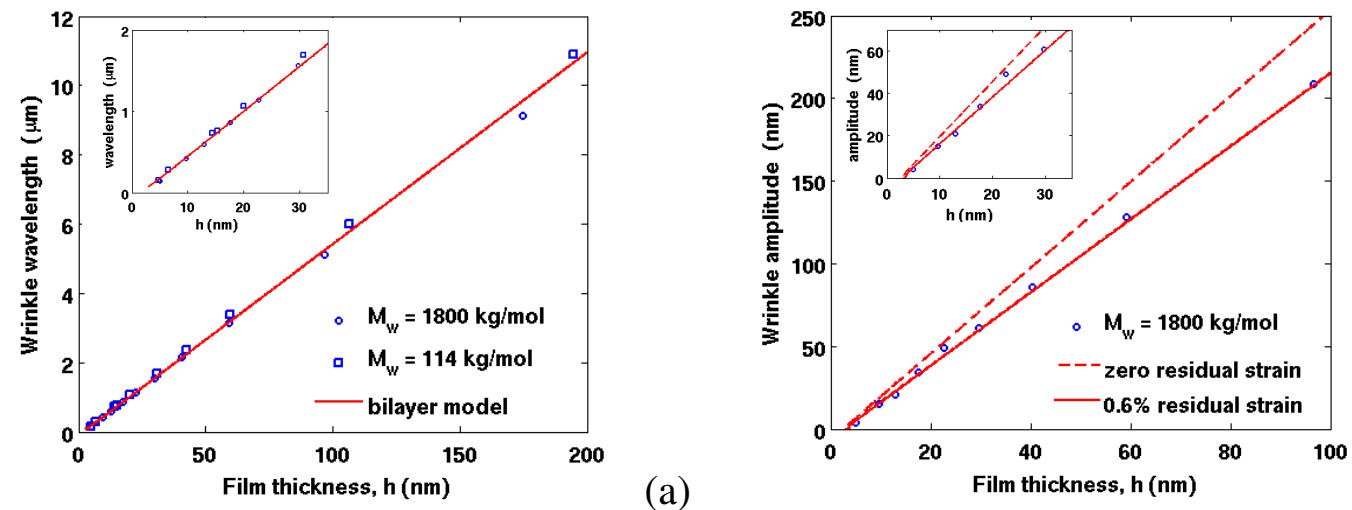

Figure 2. (a) Wrinkle wavelength and (b) amplitude as functions of the thickness of PS films, comparing experiments and the bilayer model. The insets show the ultrathin region.

\section{THEORY OF WRINKLING}

For a thin elastic film of thickness $h$ bonded to a thick elastic substrate, the critical strain for wrinkling is [1-3]:

$$
\varepsilon_{c}=-\frac{1}{4}\left(\frac{3 \bar{E}_{s}}{\bar{E}_{f}}\right)^{2 / 3}
$$

where $\bar{E}=E /\left(1-v^{2}\right)$ is the plane-strain modulus with the subscripts $f$ and $s$ for the film and the substrate, respectively. The negative sign denotes compression. When $\varepsilon<\varepsilon_{c}$, a bifurcation occurs and the stable equilibrium state of the film wrinkles in a sinusoidal form with a wavelength $\lambda$ and an amplitude $A$ as given below [3]:

$$
\lambda=2 \pi h\left(\frac{\bar{E}_{f}}{3 \bar{E}_{s}}\right)^{1 / 3}, \quad A=h \sqrt{\frac{\varepsilon}{\varepsilon_{c}}-1} .
$$


The above results are obtained as a consequence of competition between elastic strain energy in the film and that in the substrate. Specifically, wrinkling relaxes the compressioninduced strain energy in the film, but leads to an increase in bending energy in the film and strain energy in the substrate; Surface energy is completely neglected. As a result, the critical strain is independent of the film thickness, and the wavelength and amplitude are both linearly proportional to the film thickness. However, the experimental data for ultrathin films (insets in Fig. 2) shows a non-proportional behavior for both the wavelength and the amplitude, suggesting a breakdown of the conventional analysis.

For films with thickness at the nanometer scale, surface energy could be significant enough to compete with the strain energy and change the stability condition as well as the equilibrium state. As shown in a previous study [6], accounting for the surface energy in a stability analysis is equivalent to defining an effective membrane force in the conventional analysis, which includes contributions from stress in the bulk of the film and the surface stress, namely

$$
N=\bar{E}_{f} h \varepsilon+f .
$$

Therefore, comparing the two terms at the right hand side of Eq. (3) dictates when the contribution of surface stress would be important. Typically, it happens for film thickness less than $100 \mathrm{~nm}$. With the effect of surface stress, the critical strain for wrinkling becomes

$$
\varepsilon_{c}=-\frac{1}{4}\left(\frac{3 \bar{E}_{s}}{\bar{E}_{f}}\right)^{2 / 3}-\eta
$$

where $\eta=f /\left(\bar{E}_{f} h\right)$ is a dimensionless number that characterizes the effect of surface stress and depends on the film thickness. The number $\eta$ is negligibly small for relatively thick films, but becomes significant for ultrathin films. On the other hand, the equilibrium wrinkle wavelength (Eq. 2) is independent of the strain or membrane force and is thus unaffected by the surface stress. Therefore, including surface stress alone cannot explain the non-proportional wrinkle wavelengths observed for the ultrathin polymer films.

It is well known that many material properties (e.g., mass density, electrical conductivity) at the surface differ from their counterparts in the bulk. Such difference is negligible for large structures. For nanostructures, however, the surface-to-volume ratio is large, and surface effects can be significant. For thin solid films of interest here, one may assume that there exists a surface layer of different elastic modulus. Compared to the bulk elastic modulus, the surface elastic modulus can be either greater (hard surface) or smaller (soft surface). The thickness of the surface layer may vary from about one atomic spacing for crystalline materials [9] to a few nanometers for polymers [10]. In addition, a residual stress or strain may exist in the surface layer, which can be considered equivalent to uniformly distributing the surface stress (in the unit of force per length) into the surface layer of a finite thickness. Therefore, the originally homogeneous film becomes a bilayer composite with the same total thickness, and the effect of surface energy and surface stress is effectively accounted for by the presence of the surface layer.

For in-plane stretching/compression, the effective modulus of a bilayer film is

$$
\bar{E}_{f, \text { stretching }}=\bar{E}_{f}\left(1-\frac{\delta}{h}\right)+\bar{E}_{f}^{*}\left(\frac{\delta}{h}\right)
$$



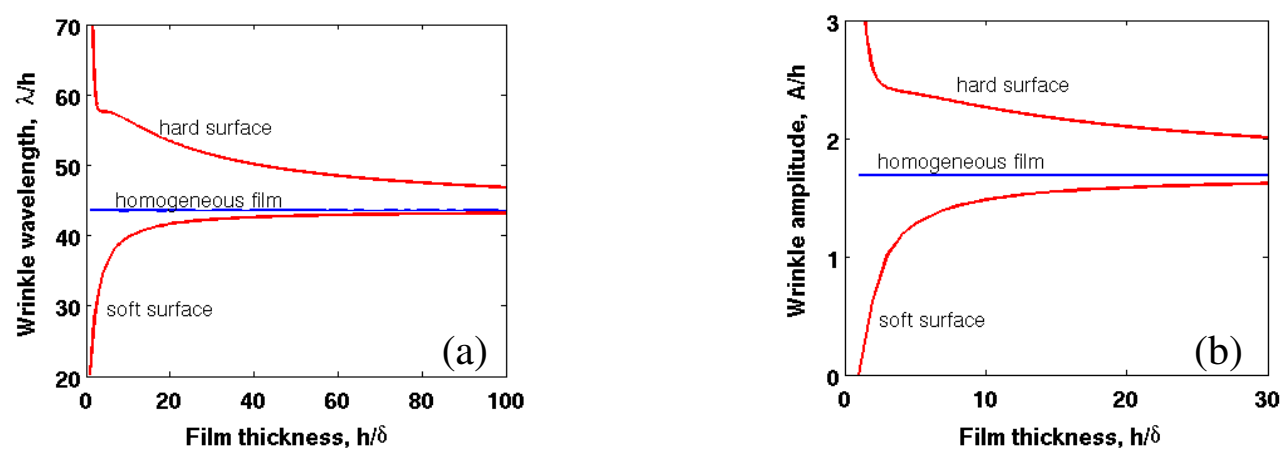

Figure 3. (a) Wrinkle wavelength and (b) wrinkle amplitude as functions of the film thickness, comparing the conventional analysis for a homogeneous film and the bilayer model.

where $\delta$ is the thickness of the surface layer, and $\bar{E}_{f}^{*}$ is the surface elastic modulus. For bending, the effective modulus is

$$
\bar{E}_{f, \text { bending }}=\bar{E}_{f}\left(1-\frac{\delta}{h}\right)^{3}+\bar{E}_{f}^{*}\left(\frac{\delta}{h}\right)^{3}+3 \bar{E}_{f}\left(1-\frac{\delta}{h}\right)\left(1-\xi-\frac{\delta}{h}\right)^{2}+3 \bar{E}_{f}^{*}\left(\frac{\delta}{h}\right)\left(2-\xi-\frac{\delta}{h}\right)^{2},
$$

where

$$
\xi=\frac{\bar{E}_{f}\left(1-\frac{\delta}{h}\right)^{2}+\bar{E}_{f}^{*}\left(\frac{\delta}{h}\right)\left(2-\frac{\delta}{h}\right)}{\bar{E}_{f}\left(1-\frac{\delta}{h}\right)+\bar{E}_{f}^{*}\left(\frac{\delta}{h}\right)}
$$

Following the same approach as the conventional analysis [1-3], the critical strain for wrinkling of the bilayer is

$$
\varepsilon_{c, \text { bilayer }}=-\frac{3^{2 / 3}}{4} \frac{\bar{E}_{f, \text { bending }}^{1 / 3} \bar{E}_{s}^{2 / 3}}{\bar{E}_{f, \text { stretching }}}-\frac{f}{\bar{E}_{f, \text { stretching }} h} .
$$

Equation (8) is similar to Eq. (4) except for the difference in the effective moduli. The equilibrium wrinkle wavelength is given by

$$
\lambda_{\text {bilayer }}=2 \pi h\left(\frac{\bar{E}_{f, \text { bending }}}{3 \bar{E}_{s}}\right)^{1 / 3},
$$

and the wrinkle amplitude is

$$
A_{\text {bilayer }}=h \sqrt{\left(\frac{\varepsilon+\eta}{\mathcal{E}_{c, \text { bilayer }}+\eta}-1\right) \frac{\bar{E}_{f, \text { bending }}}{\bar{E}_{f, \text { stretching }}}} .
$$

Figure 3(a) plots the wrinkle wavelength as a function of film thickness. For a homogeneous film ( $\bar{E}_{f}^{*}=\bar{E}_{f}$ ), Eq. (9) reduces to Eq. (2), and the wavelength-to-thickness ratio is a constant. For a bilayer film, however, the wavelength-to-thickness ratio depends on the film thickness, especially when the ratio $h / \delta$ is small. The wavelength decreases as the film 
thickness decreases for a soft surface $\left(\bar{E}_{f}^{*} / \bar{E}_{f}=0.1\right)$, and increases for a hard surface ( $\bar{E}_{f}^{*} / \bar{E}_{f}=10$ ). In both cases, the wavelength approaches to that for a homogeneous film when $h / \delta>>1$. Figure 3(b) plots the wrinkle amplitude as a function of film thickness, subjected to a constant strain $(\varepsilon=-0.02)$. Again, the amplitude-to-thickness ration is a constant from the conventional analysis. For a bilayer film with a soft surface layer, the amplitude-to-thickness ratio decreases as film thickness decreases, eventually becoming zero at a critical thickness. This critical thickness can be determined from Eq. (8) by equalizing the critical strain to the applied strain. The critical strain itself is a function of film thickness that increases rapidly as film thickness decreases for the ultrathin films with a soft surface and a tensile surface stress.

\section{DISCUSSIONS}

The measured wrinkle wavelengths and amplitudes of the PS films exhibit the general trend of decreasing with decreasing film thickness (Fig. 2). Using the measured modulus for the PDMS substrate and assuming Poisson's ratio $v_{s}=0.5$, the plane-strain modulus of the PS films are extracted from the wrinkle wavelengths by Eq. (2), as plotted in Fig. 4. For relatively thick films $(h>30 \mathrm{~nm})$, the extracted modulus is nearly constant, independent of film thickness. For both molecular masses, the plateau modulus is about $4 \mathrm{GPa}$, which corresponds to Young's modulus $E_{f} \approx 3.6 \mathrm{GPa}$ (assuming Poisson's ratio $v_{f}=0.33$ ), in good agreement with reported data for bulk PS [11] and relatively thick PS films [12]. For ultrathin films $(h<30 \mathrm{~nm})$, however, the deduced modulus drops significantly. For the thinnest film in this study $(h \approx 5 \mathrm{~nm})$, the modulus is nearly an order of magnitude lower than the bulk modulus. A similarly low modulus was estimated by Zhao et al. [13] for $10 \mathrm{~nm}$ PS films probed by a wafer curvature method.

The physical origin of the thickness-dependent modulus for ultrathin PS films can be reasonably understood with the bilayer model. According to Eq. (9), the extracted modulus in Fig. 4 is in fact the effective modulus of the bilayer for bending (Eq. 6). By assuming a soft surface layer $\left(\bar{E}_{f}^{*}=0.1 \mathrm{GPa}\right.$ and $\delta=2 \mathrm{~nm}$ ) and keeping the modulus constant for the rest of the film $\left(\bar{E}_{f}=4.2 \mathrm{GPa}\right.$ ), the predicted effective modulus is plotted in Fig. 4, which is in reasonable agreement with the experimental data. The assumption of a soft surface layer is consistent with a previous study [10] showing that, within a layer of about $1.5 \mathrm{~nm}$ at the free surface of polystyrene, the mass density is lower than that in the bulk. For thick films, the surface effect is negligible, and the effective modulus is independent of the film thickness. For ultrathin films, the presence of a soft surface layer significantly reduces the effective modulus. The transition thickness is about one order of magnitude greater than the thickness of the surface layer.

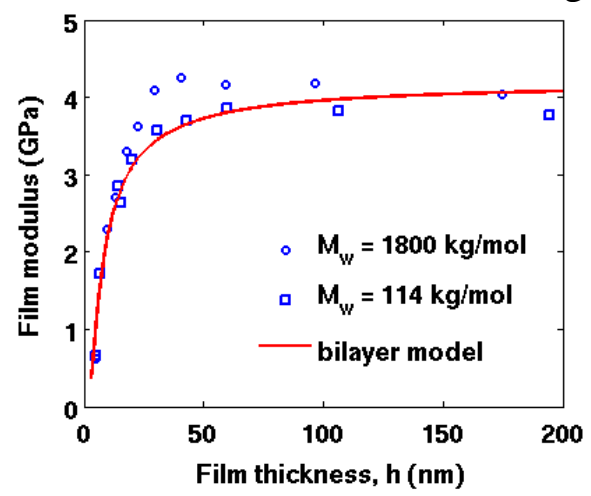

Figure 4. Effective modulus as a function of film thickness for PS films, comparing the experimental data and the bilayer model. 
Using the same parameters, the wrinkle wavelength is plotted as a function of film thickness in Fig. 2(a). The substrate modulus is taken to be the average of the two measured values. The agreement between the model and the experimental data is consistently good for the entire range of film thickness. Furthermore, the predicted wrinkle amplitude (Eq. 10) is plotted in Fig. 2(b). In addition to the above parameters, the amplitude also depends on the compressive strain and the surface stress. While the applied strain in the experiments is constant (2.5\%), there is likely a residual strain in the PS films before loading. Two curves are plotted in Fig. 3(b): one assuming no residual strain and the other with a $0.6 \%$ tensile residual strain for all films. For both cases, the surface stress is taken to be $f=0.1 \mathrm{~N} / \mathrm{m}$. Clearly, the curve with zero residual strain overestimates the amplitudes, especially for thick films. By assuming a $0.6 \%$ residual strain, the prediction of the bilayer model is in excellent agreement with the measured wrinkle amplitudes.

By comparing the bilayer model and the experimental data, a set of material properties can be deduced. For example, the bulk elastic modulus can be deduced from the plateau of the effective modulus as in Fig. 4, the thickness of the surface layer can be estimated from the transition of the effective modulus, and the residual strain in the film can be deduced from wrinkle amplitudes as in Fig. 2(b). On the other hand, the determination of the modulus of the surface layer and the surface stress is less accurate unless sufficient experimental data is available in the ultrathin region with film thickness less than $10 \mathrm{~nm}$.

\section{ACKNOWLEDGMENTS}

RH is grateful for the support by the NSF through Grant CMS-0547409 and by Texas Advance Technology Program. CMS and BV gratefully acknowledge the NIST National Research Council Postdoctoral Fellowship Program.

\section{REFERENCES}

1. H.G. Allen, Analysis and Design of Structural Sandwich Panels. Pergamon, NY, 1969.

2. J. Groenewold, Physica A298, 32-45 (2001).

3. Z.Y. Huang, W. Hong, Z. Suo, J. Mech. Phys. Solids 53, 2101-2118 (2005).

4. S.H. Im and R. Huang, Acta Mater 52, 3707-3719 (2004).

5. R. Huang, J. Mech. Phys. Solids 53, 63-89 (2005).

6. R. Huang and Z. Suo, Thin Solid Films 429, 273-281 (2003).

7. C.M. Stafford, C. Harrison, K.L. Beers, A. Karim, E.J. Amis, M.R. VanLandingham, H.-C. Kim, W. Volksen, R.D. Miller, E.E. Simonyi, Nat. Mater. 3, 545-550 (2004).

8. C.M. Stafford, S. Guo, M.Y.M. Chiang, C. Harrison, Rev. Sci. Inst. 76, 062207 (2005).

9. H. Ibach, Surf. Sci. Rep. 29, 195-263 (1997).

10. K.F. Mansfield, D.N. Theodorou, Macromolecules 24, 6283-6294 (1991).

11. J.R. Fried, Polymer Science and Technology. Prentice Hall, New Jersey, 1995.

12. L. Sun, J.R. Dutcher, L. Giovannini, F. Nizzoli, J.R. Stephens, J.L. Ord, J. Appl. Phys. 75, 7482-7488 (1994).

13. J.H. Zhao, M. Kiene, C. Hu, P.S. Ho, Appl. Phys. Lett. 77, 2843-2845 (2000). 